Rhode Island College

Digital Commons @ RIC

Master's Theses, Dissertations, Graduate

Master's Theses, Dissertations, Graduate Research and Major Papers Overview

Research and Major Papers

$6-1-2020$

\title{
Critical Care Nurses' Attitudes Towards End-of-Life
}

Ny My Tran

Follow this and additional works at: https://digitalcommons.ric.edu/etd

Part of the Nursing Commons

\section{Recommended Citation}

Tran, Ny My, "Critical Care Nurses' Attitudes Towards End-of-Life" (2020). Master's Theses, Dissertations, Graduate Research and Major Papers Overview. 373.

https://digitalcommons.ric.edu/etd/373

This Major Paper is brought to you for free and open access by the Master's Theses, Dissertations, Graduate Research and Major Papers at Digital Commons @ RIC. It has been accepted for inclusion in Master's Theses, Dissertations, Graduate Research and Major Papers Overview by an authorized administrator of Digital Commons @ RIC. For more information, please contact digitalcommons@ric.edu. 
CRITICAL CARE NURSES' ATTITUDES TOWARDS END-OF-LIFE

by

Ny My Tran, BSN, RN

A Major Paper Submitted in Partial Fulfillment

of the Requirements for the Degree of

Master of Science in Nursing

in

The School of Nursing

Rhode Island College

2020 
Abstract

Palliative care education for nurses on a critical care unit provides knowledge on pain and symptom burden management. Lack of education can detour patients away from optimal end-of-life care. A needs assessment on multiple critical care units was completed to assess views on death and dying. Initiating a needs assessment using Frommelt Attitude Toward Caring of the Dying (FATCOD) scale was utilized to identify attitudes towards caring for the dying. The goal of this project was to identify current attitudes in end-oflife care and evaluate the needs for additional education to improve patient outcomes. A total of 57 questionnaires were analyzed, two categories were observed within the survey results; nurses' attitudes towards family members of the terminally ill patients and nurses' attitudes towards caring for dying patients. Overall, the survey indicated a high confidence and positive attitude in both patient and family care. Nurses should continue to advocate and deliver optimal end-of-life patient care and reflect on their personal beliefs that may challenge caring for dying patients and their families. It is important that continuous education and self- reflection is routinely practiced. Although a consultation is optional, it is important that providers are aware of their limitations and are able to utilize available resources.

Keywords: critical care nurses, palliative care education, attitudes, caring for the dying 


\section{Acknowledgements}

I would like to thank my first reader Dr. Mock, for being patient and supportive throughout the last two years, you have been a respectable mentor and I appreciate you for pushing me to be my best, even when life got tough. To my second reader, Julia Twining, thank you for giving me the spiritual and mental support I needed to find my purpose in life, for you I am grateful for and extremely excited to work beside you as I begin my journey as a palliative care provider. To my classmates at Rhode Island College, without your laughs and positive peer feedback that boosted my confidence, this project would not be complete without your love and encouragement, thank you for the perseverance.

To my loving family, may this thesis be dedicated to you all. Thank you for helping me understand what life and death was and how to cope with both, the value of family and the gift of genuine kindness. You are my number one fans who supported me through all my adversities- thank you for being part of this life journey with me. To my dad, you believed in me when I was 14 years old and foreseen that I would change the world, I know you would be proud of me today. I will always be grateful for the impact you've had in my life; we miss you tremendously. 


\section{Table of Contents}

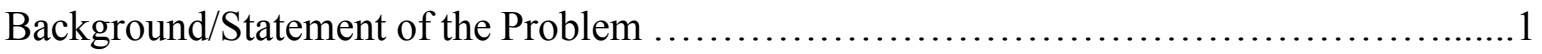

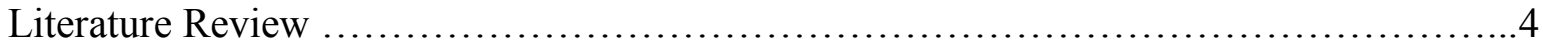

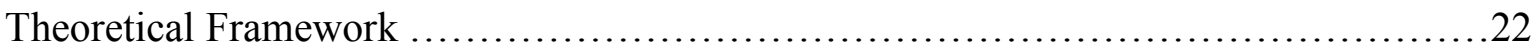

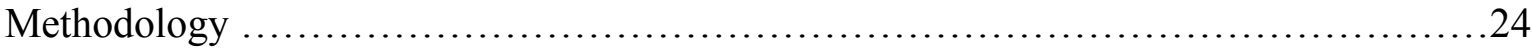

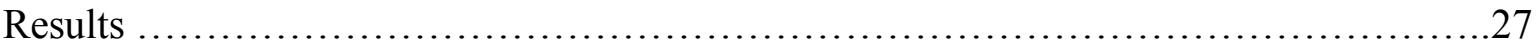

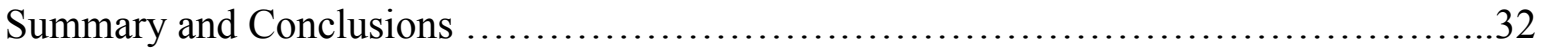

Recommendations and Implications of Advanced Nursing Practice ....................37

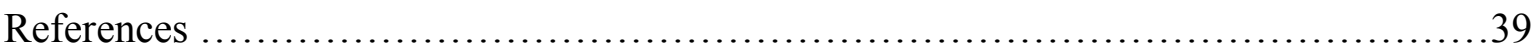

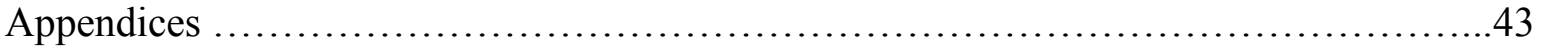




\section{Critical Care Nurses’ Attitudes Towards Caring For the Dying}

\section{Background/Statement of the Problem}

Palliative care (PC) improves the quality of life for patients and their families living with life- threatening illnesses. It focuses on pain and symptom management through physical, emotional, psychosocial and spiritual interventions. According to the Center of Disease Control and Prevention (CDC) 70\% of deaths per year are a result of serious chronic diseases, highlighting the vital role of palliative care (CDC, 2015.) Many of those deaths occur in the acute care setting, often in the critical care areas.

Palliative care services integrate the expertise of an interdisciplinary team to coordinate and address the complex needs of patients who are critically ill with a poor disease prognosis and provide ongoing support for patients and their families. National Coalition for Hospice and Palliative Care (NCHPC) defined palliative as a medical subspecialty that focuses on expert assessment and effective pain and symptom management while coordinating holistic care and support for seriously ill patients and their families. In 2002, multiple hospice and palliative care organizations developed and disseminated updated versions of Clinical Practice Guidelines for Quality Palliative Care (2004, 2009, and 2013). This guideline serves as the framework to integrate the valued principals of quality palliative care at the bedside. The National Consensus Project for Quality Palliative Care (NCP) is an initiative of the NCHPC to further define and evaluate programs and improve the delivery of palliative care in the United States.

The National Consensus Project (NCP) developed guidelines for quality palliative care services serving as the foundation for expanding an interdisciplinary team. As healthcare continues to evolve, current guidelines are continuously re-evaluated to 
provide high quality palliative care delivery. The intent of the guidelines is to improve the access for all patient populations and integrate palliative care principles and best practices into the providers routine assessment and focus on patient centered goals. The NPC identifies current evidence-based practices and educate healthcare professionals on the benefits of early palliative interventions and support the integration of high quality palliative care practices for seriously ill patients and their families.

Approximately one in five deaths in the United States occurs during or shortly after an ICU admission, with more deaths occurring in the ICU than any other setting in the hospital (Angus et al., 2004) Post intensive care syndrome includes both physical and psychological symptoms with deficits in function and cognition, therefore impairs the patient's quality of life. Aslakson et al. (2015) described palliative care as an interprofessional specialty, unlike hospice, palliative care is not based on prognosis but focuses on managing the patients' symptoms and develop an essential care plan aligned to their goals. Effective integration of palliative care during an acute health event and the diagnosis of chronic illnesses has the potential benefits to support patients and their families as well as prepare them for challenges they may encounter during and after the critical care experience.

Critical care nurses are part of the interdisciplinary team and provide an important role in palliative care discussions. Palliative care aims to improve the quality of life for patients and families impacted by chronic, serious, and life-threatening illness across the aging population. However, nurses often do not receive a formal education in this subspecialty, contributing to a lack of skills and confidence in palliative care across acute care settings (Ashley \& Fasolink, 2016.) The World Health Organization (WHO) 
recognizes palliative care as a global public health priority for patients with chronic illnesses. Multiple studies report that registered nurses may not be prepared to provide optimal care due to the lack of knowledge and their role in palliative care and hospice (Sekse et al., 2017) Congruently, nurses lack confidence in having goals of care discussions, symptom management and end-of-life care (Anderson et al., 2016.) Skar, (2010) addresses how nurses are the most valuable palliative care team member, integrating the physical, functional, social, and spiritual dimensions of patient care. Additionally, Skar noted important factors that influence the successful delivery of palliative care and concluded that the health care professionals' knowledge, attitudes, beliefs, and experiences are characteristics that impact their behavior during a clinical evaluation and treatment intervention. The results showed that having poor attitudes and palliative care knowledge may affect the patients' quality of life and deviate from their goals of care. To ensure a successful palliative care education program, a needs assessment can be completed providing targeted areas that support the ongoing educational needs of nurses and their current attitudes towards palliative and end-of-life practices. 


\section{Literature Review}

A comprehensive literature review was conducted within the years of 2010 through 2019 using key words: "Palliative Care Education”, "Critical Care Unit”, “Palliative Care Needs Assessment”, "Registered Nurse: Clinical Skills" and "Knowledge, Attitudes and Confidence". Articles were accessed via PubMed, CINAHL, MEDLINE and the Cochrane library.

The National Health Council (NHC) reported in 2014 that 133 million Americans are affected with incurable and ongoing, chronic diseases. This represents about $40 \%$ of United States total population. Additionally, the health council projected by 2020 an increase estimating 157 million will be affected with chronic diseases, with 81 million having multiple conditions. Aldridge and Kelley, 2015 conducted an analysis to identify and target "high-cost" groups. In 2011, they estimated that $10 \%$ of the $\$ 1.6$ trillion spent on medical expenses was devoted to care of individuals in their last year of life. Authors of that study concluded that health interventions to reduce health care cost should target those with long-term chronic conditions and limitations. The study concluded early treatment of palliative care for chronic and progressive diseases to lower the cost at end of life.

\section{Palliative Care in the United States}

Dumanovsky et al. 2015 comprised a multi-comparative report from previous years to track the growth of hospital-based palliative care programs across the United States. The researchers noticed hospitals with palliative care programs enhanced the quality of life of seriously ill patients in both the inpatient and outpatient setting. The status report identified hospitals with palliative programs and analyzed where gaps in 
obtaining access remains. Hospitals who provided palliative care services voluntarily register to the national data bases such as the National Palliative Care Registry, American Hospital Association (AHA) and Annual Survey and the American Census Bureau Community Survey (ACS). The National Palliative Care Registry reported institutions that offered palliative services within the United States. The survey categorized each institution by region, hospital ownership and size. The ACS gathered national demographical data for each state.

Sixty-seven percent of eligible hospitals that met inclusion criteria reported to have palliative care programs at their institution. Researchers assigned proficiency grades to each state based on the effectiveness of their palliative care programs. States were scored based on their participation in palliative care programs. States with over $80 \%$ participation in palliative programs were graded an $A$, and consequently, states with less than 20\% participation received an $F$ and a revision plan. During the fiscal years of 20122013, 981 (62\%) palliative care programs were identified through the National Palliative Care Registry, 610 (38\%) were identified through web searches or telephone surveys with a trained interviewer. The states were grouped into categories of hospital size, tax status, region and association with an accredited residency training program. Seventeen states were awarded an $A$ with over $80 \%$ of participating palliative care programs, 7 states were graded a $D$, surprisingly no states that met inclusion criteria has lower than $20 \%$ of palliative participation. Hospitals with less than 50 beds were excluded due to limited capacity and staff to provide palliative services. The survey addressed the hospital patient capacity, results separated institutions with more than 300 beds compared to institutions with less than. Upon review, $90 \%$ of hospitals with more than 300 beds were 
found to have a palliative care program compared to hospitals less than 300 beds, an average of $56 \%$ offered palliative programs. Researchers also noted that hospital ownership was a strong variable for offering palliative care services and became dependent on their patient population and profits. Not-for profit and public hospitals were found to offer more participation of palliative services compared to for-profit hospitals. The ACS recognized New England as the highest region of participation in palliative services, the lowest region was located in the southwest and southeast of the United States. In addition, geographical and demographical data of hospitals with accredited residency training programs were observed to have offer more palliative care services compared to hospitals without medical residency training programs.

A recent report demonstrated improvement in palliative care outcomes over the past 16 years, illustrating a steady increase since the original report in 2001. This fiscal year survey did not report any states with less than $20 \%$ of palliative services, indicating a growth of programs within the United States. Dumanovsky et al. (2001) observed the lack of access for palliative programs was often related to hospital size, ownership and region. Included in this study, the Institute of Medicine suggested opportunities to improve access to palliative care by incorporating palliative care training, reporting accredited programs, evidence-based investment in palliative research to measure the quality of care for patients that are eligible for palliative service.

\section{Palliative Care in Critical Care Areas}

Anderson et al. (2016) performed a multicenter survey in five hospital intensive care units (ICU), to explore the perspectives of ICU nurses and their involvement in palliative care communication. This was an initiative to explore the needs and 
perspectives of nurses on palliative care in the ICU. Surveys assessed the nurses' perspective and frequency of participating in palliative care discussions, confidence in performing palliative care tasks and barriers to their involvement in palliative care discussions. ICU nurses responded to the 46 -item survey (mean $=33 \%$ ), overall $(95 \%)$ reported that discussions between families and clinicians about prognosis and goals of care were very important to the quality of care for ICU patients. About $88 \%$ of bedside nurses felt that prognosis and goals of care discussions between the families and clinicians was very important to a patient's quality of care. The infrequency of nursing involvement results varied, $75 \%$ reported engaging in goals of care discussions often with physicians. An average of $24 \%$ reported a lower participation in attending family meetings, and 19\% had active participation in family discussions. The highest degree of confidence in performing palliative care tasks reported $34 \%$ of nurses were "very confident" in conveying the need for a family meeting to a physician. Respectively $41 \%$ of nurses felt "somewhat confident" when ensuring that patients and families receive palliative care when needed. Anderson et al. (2016) correlated night shift nurses to feel "not confident" in tasks related to family meetings compared to day shift nurses. Authors suggested a lower confidence in night shift nurses may relate to infrequent palliative care discussions during the offshifts. Limitations in this study included a large number of nurses who did not respond to the survey may have affected the results. However, their large sample size was suggestive as a representative sample of ICU nurses. Other findings include insufficient education of nurses, lack of collaboration with the physician and participation in goals of care discussions. The authors' findings reinforce their 
previous findings and disseminated the value of bedside nursing engagement as an important component in high-quality palliative care delivery in the ICU.

Anderson et al. identified a need for formal education in palliative care and skills to optimize therapeutic communication techniques for nursing practice and effectively provide emotional support to patients and their families while sustaining their own personal well-being. In addition, researchers suggested that education will increase the awareness to be actively involved in optimal palliative care to benefit these patients and their families. Clinician support for mental and emotional health was recommended due to the continuous use of emotional practice coupled within a highly intensive work environment. The authors concluded that ICU bedside nurses have an important role in palliative care communication. There is a need for further interventions to enhance their involvement and confidence in palliative care which may be improved through education and emotional support.

\section{Role of The Interdisciplinary Team}

Beggs et al. (2014), comprised a literature review about the role of the interdisciplinary team caring for dying patients in the ICU. The aim of this review was to advocate the use of collaborative team members to improve the patient care experience in the ICU. A collaborative interdisciplinary team was described as members from various health disciplines with specialized knowledge and skills who care for the same patients, using shared planning and goals to systemically communicate and coordinate care (Schmitt et al., 2001). The members of an ICU team include physicians, residents and fellows, nurse leaders and staff nurses, pharmacists, social workers, respiratory therapist, 
physical and occupational therapy, and chaplains. The role of the collaborative approach is utilized towards effective treatment and decision making for the ICU patient.

Researchers identified the key activity for collaboration teams is comprised of physicians and nurses working together and sharing responsibility for problem-solving and decision making and formulate and carry out care plans for patients. Beggs et al. reviewed a study on grounded theory and examining the process of a successful collaboration between the physician and nurse. The study discussed the relationship between disciplines and found that "working together" and focusing on the patient and recognizing the important roles of each team members through knowledge sharing and communication was the core value when taking caring for dying patients in the ICU. The study concluded that the use of a collaborative interdisciplinary team would lead to better patient outcomes and a positive work environment, however barriers to collaboration need to be assessed and identified.

Researchers recognized the success of team care collaboration, resulting in improve care and patient outcomes, including the end-of life support for the dying patient and their families. Additionally, Beggs et al. (2014) identified the need for specific interventions at the unit and individual level. The unit interventions included policies valuing team care, objectives for shared responsibility and interdisciplinary leadership and committee structure. At the individual level, interventions included education in a clinical setting and effective interpersonal interactions. Upon comparison of this literature review, the collaboration of a multidisciplinary team is a key component of providing optimal palliative care delivery. 
Puntillo (2001) characterizes the role of a critical care nurse as an integral member of the ICU team caring for the dying patient. Based on these Puntillos' findings, the role of nurses should not be minimized, undermined, or ignored and valued for their expertise and commitment to caring for the dying ICU patient. In addition to providing end-of-life care, nurses have a key role in interacting with families of these dying patients. Researchers noted that high quality patient care requires the collaborative commitment between the physician and the nurse by improving their communication.

Lilly (2000) conducted a quality improvement project that implemented an interdisciplinary approach to improve communication with ICU families and patient outcomes. The communication intervention consisted of family meetings lead by the physician, nurse and house officer. The goal of the project was to proactively identify clinical milestones and goals of care that improve the patient overall prognosis or to consult palliative care earlier in patients who meet palliative care criteria or chose conservative care. The intervention created an opportunity for patients to be introduced to palliative care when appropriate to their goals of care. A four year follow up was conducted and the results indicated a reduction in average length of stay and reduced mortality rates when the communication intervention between the physician and nurse was implemented. In discussion, Lilly et al., recommended the use of interdisciplinary teams with the collaboration of adjunct teams such as palliative care to improve patient outcomes. In addition to early initiation of palliative care consults, evidence suggested savings on unnecessary medical expenditures, improved communication between the patients, families and interdisciplinary team and improved family and staff satisfaction aligned with their goals of care. 


\section{Knowledge and Attitudes Towards Care of the Dying}

Palliative care is traditionally viewed as the intense care of patients who are close to death. A descriptive cross-sectional exploratory study (Ayed et al., 2015) assessed the nurses' knowledge and attitudes towards palliative care. Initially a pilot study was conducted to test the interventional instrument for validity with ten nurses on the medical and surgical units to determine the clarity of questions, effectiveness of instructions, completeness of response sets, timing for study and data collection. The revised study was completed and distributed to four hospitals in the North West Bank region of Palestine. The goal is to gather information on nurses and assess their knowledge and attitudes in palliative care. Inclusion criteria included nurses with full time employment working in the ICU, emergency room and medical and surgical units.

The researchers developed a questionnaire, assessing the socio-demographic variables including age, gender, hospital, certifications, job position, department and work experience including training for critical ill patients. To assess the nurses' palliative care knowledge, a group of palliative care questions were provided. The correct number of answers were totaled as a percentage and classified as poor knowledge, fair knowledge and good knowledge. Attitudes were assessed using the 5-item Likert scale, ranging from strongly agree to strongly disagree. The highest possible score for the attitude assessment was a 60 and attitudes were categorized as good, moderate and poor.

Data was collected at four different hospitals and 96 nurses participated in the study. Demographic data was collected for baseline characteristics of the sampled population. About two-thirds (average $=74 \%$ ) of participating nurses were within the age of 20-30 years, $19.8 \%$ fell into the age range of $31-40$ years and $6.3 \%$ were older than 41 
years of age. Sixty-six male and 30 female nurses participated. The demographics also included their level of education, $58.3 \%$ of the nurses held a bachelor's degree, $36.5 \%$ had their diploma or less and 5.2\% obtained their masters. Data was distributed to four different units, $30.2 \%$ worked on the medical unit, $15.6 \%$ on a surgical unit, $26 \%$ in the ICU and $28.1 \%$ were from the emergency department. Respectively, $56.2 \%$ had less than five years of nursing experience, $59.4 \%$ of the nurses answered that they had training in palliative care. However, $57.9 \%$ of the nurses had training of one week or less, comparatively $42.1 \%$ reported having more than one week of palliative care training. Authors formatted 14 educational questions to assess the nurses' knowledge in palliative care; $45.8 \%$ had poor knowledge in palliative care, $33.3 \%$ had fair knowledge and $20.8 \%$ were rated to have good knowledge. Attitudes of nurses towards palliative care summarizes that $63.5 \%$ of nurses disagree that palliative care is given only for dying patients. More than half (56.7\%) of nurses disagree that they should not withdraw their involvement with the patient. On the contrary upon attitude assessments, $56.3 \%$ of nurses agree that patients will benefit from verbalizing their feelings; $59.4 \%$ agreed that families should be involved in the patients physical care at end of life; $43.7 \%$ of nurses admitted to be afraid of becoming friends with chronically ill patients while $47.9 \%$ found it difficult to form a relationship with a dying patient. Prospectively, $52.1 \%$ of nurses felt uncomfortable if they entered a room and found the patient crying. The study reported that $56.2 \%$ of the nurses possess a moderate attitude in palliative care, giving that confidence affects their attitudes.

In conclusion, the authors recommended collecting the baseline knowledge and personal beliefs before establishing educational programs. The study also suggested that 
the lack of knowledge may be associated with the lack of palliative care units in Palestine. In addition to lack of palliative care experience, palliative education was not incorporated in nursing curriculums, explaining their limited skills. These findings indicated that nurses are not consistently knowledgeable in palliative care, therefore a emphasize for a need of standard palliative education will enhance their knowledge and attitudes. Ayed et al., 2015 included a recommendation to incorporate palliative care education in academic nursing curriculums.

\section{End-of-Life (EOL) Education}

The Archstone Foundation (2008) launched an End-of Life initiative to change the dying culture in California. The foundation aims to build a strong palliative care program that improves patient care, management of symptoms and better quality of life by increasing education and training of nurses, physicians and social workers in palliative care. Education was provided through the End-Of-Life Nursing Education ConsortiumCritical Care (ELNEC-CC) program. The curriculum was developed using previous research studies in end-of-life care to implement evidence-based care, this training program was specific to critical care nurses.

Ferrell et al. (2010) emphasized the important role of critical care nurses in improving the experience of the dying patient. They identified the lack of education in providing end-of-life care as an obstacle for nurses which impacts the delivery of palliative care. A group of critical care nurses developed the ELNEC-CC curriculum, using the American Association of Colleges of Nursing: A Peaceful Death document as the framework. Teaching materials and $E O L$ education included: an overview of palliative nursing care as it related to critical care interventions; pain and symptom 
management; loss, grief, bereavement, communication, ethical issues, final hours, and achieving quality palliative care.

Eighty-seven California nurses attended the training program. Participants were asked to complete a preassessment survey on their current perspectives on palliative care and how end-of-life/palliative care is provided within their institution. The program was conducted in two and a half days, including an overview of palliative nursing, didactic lectures, specific critical care discussions case studies, role playing and use of videos to demonstrate teaching strategies. At six and twelve months post training, nurses were reevaluated on the program's effectiveness. This study evaluates the effects of formal palliative care education for registered nurses on improving patient outcomes aligned with their goals and end-of-life experience. Participants of the study reported benefits and effectiveness of the ELNEC-CC training by improved scores in areas: teaching palliative/end-of-life content; effective palliative education provided within their current institution and the overall helpfulness of the ELNEC-CC program. Effective examples of program dissemination reported by nurses included; policy and systemwide changes, improved bereavement care and professional nursing education, revising end-of-life orders for patients in the ICU, educating the interdisciplinary team on new comfort care orders, symptom management, terminal ventilator weaning, empathic and cultural considerations with a dying patient, implementing palliative rounds/increase palliative care consults, providing pastoral care services, condolence cards and offer bereavement services. ELNEC-CC are able to educate faculty and nurses about palliative care and reviewing pain and symptom management through online modules. Barriers of this program training included lack of time management, minimal financial support, staff 
availability, lack of administrative support and poor attitudes towards death and lack of knowledge of palliative care reported by the interdisciplinary team as a whole. The study concluded the essential role of critical care nurses in palliative care. The ELNEC-CC program is a successful educational effort that addresses the need for improved education to existing curriculums geared towards critical care nurses. The contributors who developed the ELNEC-CC identified the lack of knowledge as an obstacle for health care professionals. Therefore, education in palliative care topics are urgently needed.

\section{Development of Needs Scale for Palliative Care}

Nurses play pivotal roles on palliative care teams as they possess clinical skills, patience, empathy and the ability to create a rapport with the patient and families. Chang et al. (2015) developed a correlational research study of an educational needs assessment that evaluates the needs for curriculum content and planning for goals of care related to palliative care. The purpose of this study was to identify gaps in knowledge, develop a supportive curriculum, identify resources and guide the implementations of new policies. It was designed to determine the content needs for palliative care education for nurses at a medical center in Taiwan and perform a factory analysis for curriculum needs for education. Authors developed a 42-item instrument that consisted of content needs outlined by advanced courses of the Taiwan Oncology Nursing Society, core classes by End-of-Life Nursing Education Consortium (ELNEC), content on basic palliative care training included domestic and foreign research. Responses were scored on a 5-point Likert scale from 5 (extremely necessary), 4 (strongly needed), 3 (needed), 2 (not needed), and 1 (extremely unnecessary); scored results ranged from 42 to 210. A high score is indicative of needed palliative care content. The validity index of this instrument 
tool had a Cronbach reliability score of 0.98 . The study used a random cluster sampling approach to survey all nurses in a Taiwan medical center.

Chang et al. (2015) distributed the questionnaires at morning conferences and recruited head nurses to identify absentees. There was a high response rate of 600 $(97.72 \%)$ returned surveys was over a 3-week period. The survey included sociodemographic variables such as age, gender, education level, religion, nursing role, work experience, the unit they worked on. Additional information asked if they were members of the Taiwan Oncology Society and Taiwan Association of Hospice Palliative Nursing, have had previous experience in palliative care and care for terminal patients and their families, former participation in palliative workshops and their current willingness to participate in palliative care classes. The study used an exploratory factory analysis to identify content needs for an in-service education and examine the correlation between the content factors. Results was concluded from 6 major program needs for inservice education. Factor analysis identified handling of symptoms and pain relief, ethical issues and teamwork, preparation and care before death, concept of palliative care, communication and counseling, and cultural and spiritual factors and considerations. These findings were similar to other studies indicating a need for additional palliative care education and were summarized into 4 categories: (1) physiological management of disease and pain symptoms; (2) psychological emotional support for the patient and family members; (3) social problems facing the patients and (4) spirituality: patients' belief, attitudes, customs and mourning rituals after death (Fernandes, 2009).

Researchers acknowledged pain assessment and management was found to be the highest need for in-service education. Pain has shown to have a negative impact on the 
patients' quality of life therefore nurses must appropriately assess the patients pain to meet their basic physiological needs for improve their quality of life. Additionally, respiratory difficulty, constipation, and nausea was also reported as leading physical symptoms among terminal patients (Hu et al., 2004.) Ethical and teamwork issues were identified as an educational need to ensure the goals of care aligned to the patient's preferences. Examples of ethical concerns described in this study include, disclosure of illness, nonmaleficence in life support systems for terminal illnesses and appropriateness for Do Not Resuscitate (DNR) status.

Compassion fatigue is a natural consequence that is a gradual progression caused by repeated exposure to chronic stressors (Jenkins \& Warren, 2012.) Chang et al. (2015) characterized nurses who experience a psychological impact and compassion fatigue with death and dying. However, nurses must acquire the stoic role to guide families during their grief and loss. This trait is important when families are overemotional and unable to rationalize beneficial outcomes. Within the nursing role, this chronic stress can be overwhelming.

Chang et al. (2015) disseminated areas of knowledge deficit in preparation and care before death as the third factor of education needs. The role of the nurse includes preparing the family for death, management of pain symptoms and maintaining a calming environment. Together these efforts provide a patient-centered care plan. An in-service program on death preparation is important for nurses to provide accurate and supportive resources for patients and their families. Within the healthcare continuum, it is important to treat the patients physical, psychological, spiritual and religious needs as a whole. The author concluded that nurses are primary providers across all healthcare settings and 
emphasize the importance of effective communication skills that is necessary in building palliative care programs.

Limitations of this study included a sample size limited to one hospital. The study utilized a single instrumental survey that served as gap analysis identifier, however, the instrumental tool was considered highly reliable $(\alpha=0.98)$. The study recommended implications for practice that included guidance and planning for palliative care instructions, administrative support, design of evaluation tools, continuing education and roles of the interdisciplinary team. In conclusion, the study addressed barriers through an exploratory factor analysis to develop a palliative care curriculum.

\section{Educational Intervention Using FATCOD Scale}

The increasing number of chronically and terminally ill patients justifies the need for palliative care education to accommodate and care for this specific population. Frommelt (2003) analyzed the effects of palliative care education programs and nurses' attitudes towards caring for dying patients. In 1991, Frommelt originally implemented an education program that assessed the nurses' attitudes towards caring for terminally ill patients and found that nurses who participated in the education program reported a significant improvement in their attitudes towards dying patients.

Frommelt (2003) replicated the same study and included a variety of professionals on a college campus rather than just nursing staff. The purpose of this study was to compare the relationship of demographic variables with attitudes toward caring for the terminally ill and their families. The author hypothesized those who participated in the experimental group will have a positive attitude towards caring for the terminally ill and their families. Frommelt (2003) proposed that personal values and attitudes affect the 
outcome of cognitive learning, therefore incorporating both cognitive and affective learning strategies should be included in the education program. The study was a quasiexperimental design with convenience sampling, results were compared between the experimental and the control group.

The education program was developed and included an overview of categories related to loss, grief, death, dying and bereavement, different teaching styles were adapted to accommodate all learning styles. Students in the experimental group were selfselected and enrolled in a 15-week education course, this course was offered for three consecutive semesters for appropriate sample size. The study utilized the Frommelt Attitude Toward Care of the Dying (FATCOD) developed by Frommelt, 1988, the tool valued a high reliability score of 0.93 . The attitudes of students measured by the FATCOD scale, analysis of variances and covariances was performed using an analysis of variance (ANOVA) and analysis of covariance (ANCOVA) to authenticate the accuracy of the controlled variables.

Results of the study aligned and confirmed the authors hypothesis: those who participated in the experimental group will have a positive attitude towards caring for the terminally ill and their families. A significant change in attitudes were found in the experimental group, in comparison, the control group that did not participate in the education program had no change in its results. The demographic data did not demonstrate any significant effect on the student's attitudes, however participation in a structured educational program on death and dying supported the hypothesis. In the posttest response guiding future studies, students identified the instructor and articulating personal examples of death and dying was favorable in the educational program. Overall, 
the FATCOD scale was found to be a valid and reliable tool to measure attitudes towards caring for the terminally ill and their families.

\section{Summary}

National reports monitored the growth of palliative care programs across the United States. The status of hospitals with palliative care programs who is registered in the AHA and ACS enhance the quality of life in both acute and chronic disease trajectories. Hospitals that had a palliative care program were rated on an alphabetical scale in relation to their participation. Programs that were graded an $A$ had achieved over $80 \%$ of palliative care program participation, states with less than $20 \%$ participation was awarded a $F$. The ACS recognized that New England was the highest participant to have palliative care programs, comparably southwest and southeast regions was noted to have the lowest program participation. There has been improvement in the palliative care program involvement, in the current fiscal year, there were no reports of any state to have less than $20 \%$ of palliative care participation, indicating the growth of programs within the United States.

Researchers identified that ICU teams include the collaboration of physicians and nurses and are part of the interdisciplinary team. Beggs (2015) concluded that the collaborative interdisciplinary team would lead to better patient outcomes and a positive work environment. Puntillo identified the role of a critical care nurses to be an integral member of the interdisciplinary team. At end-of-life, nurses are faced to interact with families and dying patients therefore improved and continuous communication will help proactively identify clinical milestones and goals of care that improves the patient

experience. Family meetings serves as an educational checkpoint to address current 
issues and future patient care planning. Given that only $56.2 \%$ of nurses possess a moderate attitude in palliative care, it is suggested that lack of knowledge or experience in palliative and end-of-life care may limit skills and provide less than optimal patient outcomes (Ayed et al. 2015.) The Archstone Foundation (2008) developed a strong palliative care program that improves patient care, management of symptoms and better quality of life by increasing education and training nurses, physicians and social workers in palliative care. Education was provided through ELNECC-CC programs and used evidenced based research to implement training programs specific to critical care nurses. Ferrell et al. (2010) emphasized the important role of critical care nurses and improving the patient care experience of dying patients and their families. The contributors who developed the ELNEC-CC identified lack of knowledge as an obstacle for health care professionals. Therefore, education in palliative care topics are urgently needed. 


\section{Theoretical Framework}

The theoretical framework for this project is based on Mezirow's Transformative Theory of Adult Learning (1997). Mezirow describes how people develop and use critical self-reflection to frame their own beliefs and experiences over time. The transformation in an individual's frame of reference is compounded by associations, concepts, values, feelings and responses (Mezirow, 1997.) Humans transform their frame of reference through critical reflection and the assumptions of their personal interpretations, beliefs and habits of mind or points of view. Transformative theory follows four processes of learning: elaborate existing points of view; establish new points of views; transform our point of view and becoming aware and critically reflective. This process involves transforming frames of reference throughout critical reflection of personal assumptions, validating contested beliefs by experience, taking action on one's reflective insight and assessing them. Transformative learning stimulates the accepted knowledge to think autonomously and to perform independently from personal experience, resembling a competence model (Renigere, 2014.)

Transformative education is complementary to nursing through learning outcomes, gaining knowledge and personal experiences to increase sensibility and ethical responsibility. The goal to is to understand personal actions and points of views that drive decisions by exploring the values and knowledge to increase autonomy and potential biases. Mezirow addresses the shift of consciousness and challenges of personal viewpoints and its effects to critical decision making.

Critical care nurses experience end-of-life care in variable ways, leading to varied and inconsistent patient goals. Assessing and changing current frames of reference 
regarding palliative care is essential to provide effective patient-centered care interventions for seriously ill adults. Palliative education is purposeful in shifting the nurses' point of view and support deliberate self-reflection on their current knowledge and practice. Educating nurses in palliative care increases their clinical knowledge to understand pain and symptom management, goals of care, advanced care planning and end-of-life care (NCHPC, 2001.) Using this reflective theory as a foundation for education will provide a balance between personal emotions and knowledge and support nurses to explore an effective plan of care with and for the patients (Yorks and Sharoff, 2001.)

Mezirow's Transformative Learning Theory defines the personal challenges of nurses and addresses the basis of ethical dilemmas they encounter. Essentially, providing accurate palliative care education to critical care nurses will identify outdated frames of reference and shape attitudes and beliefs to provide positive measures of self-reflection to improve patient care. 


\section{Methodology}

\section{Purpose}

The purpose of the study is to perform a comprehensive needs assessment of the current attitudes and knowledge of critical care nurses on palliative care.

\section{Sample/Setting}

This project was completed on a 247-bed acute care, teaching hospital in the northeast, in three different critical care areas: the Cardiac Care Unit (CCU), Specialized Intensive Care Unit (SICU) and the Medical Intensive Care Unit (MICU.) These critical care areas include 58-beds for patients requiring high-acuity level of care. A nonprobability convenience sampling plan was utilized. Projected sample size was registered nurses $(n=50)$ who voluntarily consented to participate in the survey. Inclusion criteria for participants was comprised of registered nurses employed on a critical care unit, English speaking, and able to complete a self-reported questionnaire. Exclusion criteria included nurses with per diem status and non-critical care nursing staff. The researcher selected participants meeting inclusion criteria through convenience sampling on all critical care units. To avoid sampling bias with convenience sampling, a representative and generalizable population of eligible participants from multiple inpatient critical care units were recruited.

\section{Procedures}

This palliative care needs assessment project was approved by the all three critical care area managers and clinical nurse educators. Permission from Lifespan and RIC's IRB was granted. The survey tool used in this study was the Frommelt Attitudes Towards Care of the Dying Scale (FATCOD). The FATCOD was created and copyrighted by 
Katherine Frommelt in 1988. FATCOD is comprised of 30 statements to evaluate the current attitudes of nurses in caring for the dying patient. The statement addresses the nurses' attitudes toward the dying patient and attitudes toward the patient's family.

The FATCOD survey items were scored on a Likert 5-point scale: 1 (strongly disagree), 2 (disagree), 3 (uncertain), 4 (agree), and 5 (strongly agree). Frommelt designed the statements to include both negative and positive formats to rule out any outliers. Scores range from 30-150, with higher scores indicating a positive attitude. (see Appendix A) for tool. Questions were reviewed by course faculty, critical care clinical nurse educator and a palliative care team member for validity and comprehension. Advertisement and reminders of the survey were sent out via flyers to all critical care areas two weeks prior to project implementation. Advertisement included goals of the project, learning objectives, start and end date of questionnaire and the researchers contact information.

All surveys remained confidential and secured by this author for study purposes. Surveys were collected two weeks within the return deadline that will be posted prospectively. A reminder in the units' weekly newsletter were sent out before the survey deadline.

\section{Measurement/Data Analysis}

All demographic data and survey results were reported as descriptive statistics. The questionnaire was analyzed to identify the attitudes of nurses on different critical care areas. The data collected was measured on a Likert 5-point scale. Total scores and trends were compared and identified for further palliative care planning purposes. 


\section{Desired Outcomes}

Ideal outcomes of this project were to identify the needs of the critical care nursing staff in palliative care knowledge and attitudes toward death and provide a gap analysis of specific education needs.

\section{Ethical Concerns}

Ethical considerations include informed consent of the participants. Participation in the study was voluntary and will free of any coercions. All data measured and collected is confidential with no identifying information was collected and or and survey results were reviewed only by the author. Diversity issues for this project include but are not limited to, participants diverse knowledge and beliefs of palliative care, religious and ethical views and cultural practices and differences as they relate to end-of-life.

\section{Organizational Factors}

Approval from the critical care unit managers and clinical nurse educator was obtained prior to project implementation. (see Appendix B). The clinical nurse educator and unit managers supported the project. It is noted that the project implementation will benefit the nursing staff in planning and design of a critical care palliative education.

\section{Evaluation}

The critical care nurse palliative care needs assessment was measured by the FATCOD survey. The survey findings will be shared with the nursing leadership in the critical care units and the palliative care team in the hospital. Additionally, the results will be disseminated as a poster presentation in all critical care area breakrooms. The project findings will be presented at the Rhode Island College MSN Major Project Poster Presentation in May 2020. 


\section{Results}

A total of 57 completed questionnaires were analyzed. All participants worked nurses on a critical care unit and no additional demographical data was collected to maintain confidentiality. The FATCOD survey was scored on a Likert 5-point scale: 1 (strongly disagree), 2 (disagree), 3 (uncertain), 4 (agree), and 5 (strongly agree). Two categories were observed within the survey results; nurses' attitudes towards family members of the terminally ill patients and nurses' attitudes towards caring for dying patients. The questions were formatted as positive and negative statements and require the nurses' opinion and current attitudes towards caring for dying patients and their families. When asked "giving nursing care to the dying person is a worthwhile experience" $63 \%$ responded with strongly agree. When asked "I would not want to be assigned to care for a dying person, 65\% responded with strongly disagree. It is indicative that nurses are comfortable with caring for the dying patient. Survey questions that were formed as negative statements had correlating lower means, compared to positive statements that yielded a higher mean indicating a positive response rate.

Twenty questions (Table 1) were identified as patient care related items. The highest mean score of confidence in patient related item asked if nurses should permit a hospice patient with flexible visitation hours, $77 \%$ and $21 \%$ responded with strongly agree and agree respectively with no negative responses. The lowest mean question asked nurses if they believed if patients should not be allowed to make decisions about their physical care, $84 \%$ and 14\% (2.30 \pm 0.61$)$ answered disagree and strongly disagree, therefore affirms nurses practiced patient center care and patient autonomy and are comfortable with patient advocacy. Ten questions (Table 2) were identified as family 
related items, these questions are asked on the behalf of caring for families of the dying patients. The highest mean asked if families need emotional support to accept the behavior changes of the dying person, $60 \%$ and $40 \%$ had a positive response rate of strongly agree and agree. The lowest mean was reflected as a negative statement and was illustrated a high response rate in regards to comfortability, nurses were asked, was it difficult to form a close relationship with the family of the dying person, 93\% responded that nurses disagree the difficulty of forming a rapport with families, while $7 \%$ was unsure, indicating that the nurses are able to provide families with comfort and support. Among these positive responses and general consensus of palliative knowledge and attitudes, "unsure" responses were obtained indicating areas of potential improvement. Six questions (Table 3) were selected that had highest mean of "unsure" responses. Further implications for this study were identified based on the high responses of unsure answers. Question12 had the highest response of unsure answers, with a mean score of 3.7 asking if the family should be involved in the physical care of the dying patient. It can be hypothesized that nurses may be uncomfortable including family members into their patient care practices. Question 3 and 8 measured levels of confidence and belief. Question 3 asked, I would be uncomfortable talking about impending death with the dying person. No surveyors answered, "strongly agree" however, 14\% responded with “unsure”, suggesting some nurses may be uncomfortable talking about death. Question 8 corresponds with the value of ones' belief, asking if they would be upset if their dying patient they were caring for gave up hope of getting better, conflicting results indicated that $14 \%$ was "unsure" if the patient's mindset determines their prognosis, while $81 \%$ responded with "disagree" and "strongly disagree." The 
connection between belief and perseverance reflects the nurses' attitudes about death may need to be further explored. These "unsure" responses were evaluated as opportunities for growth in experience, confidence, communication, belief system, delegation and support.

\section{Table 1}

\section{Mean of FATCOD Questions: Patient Related Items}
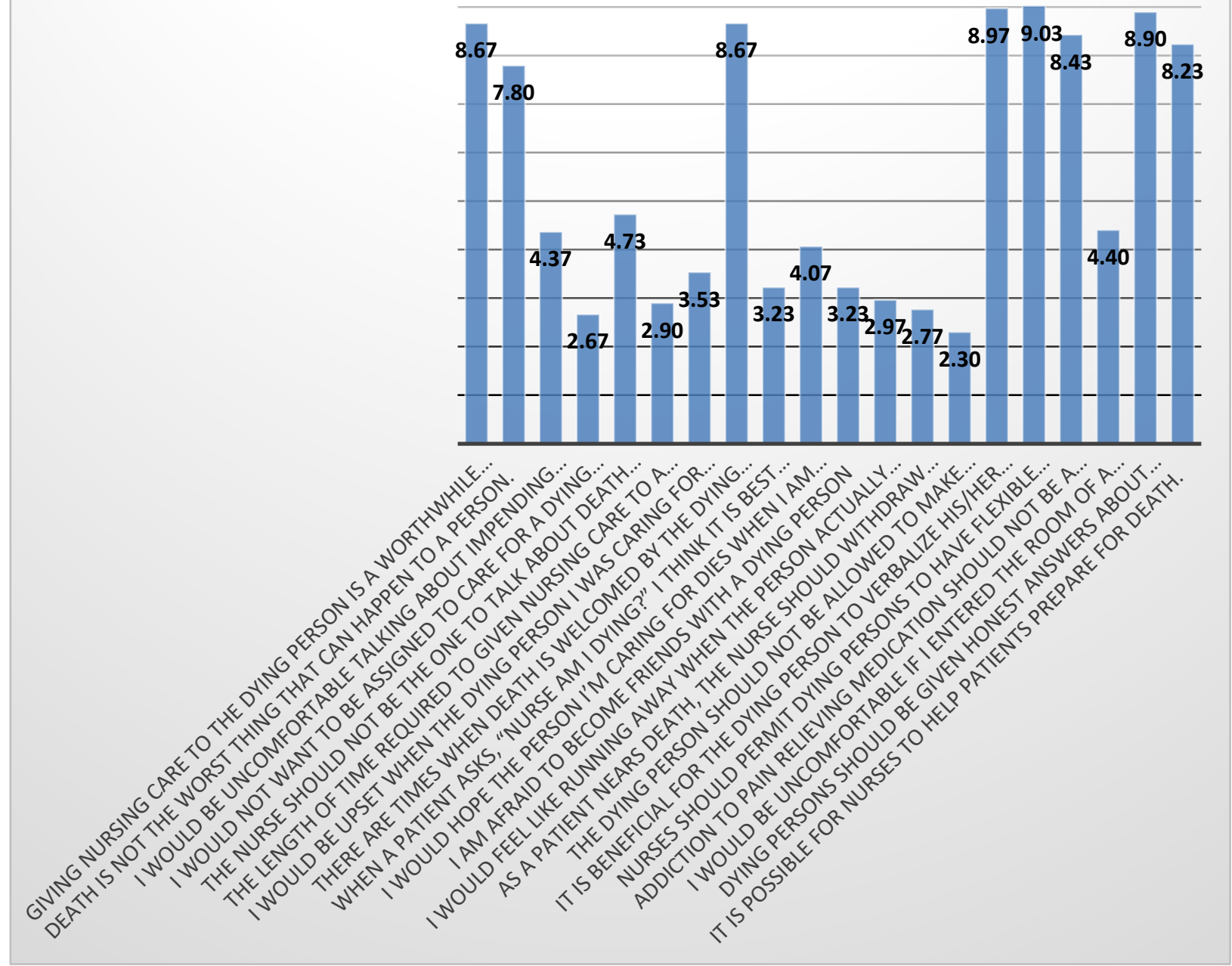
Table 2

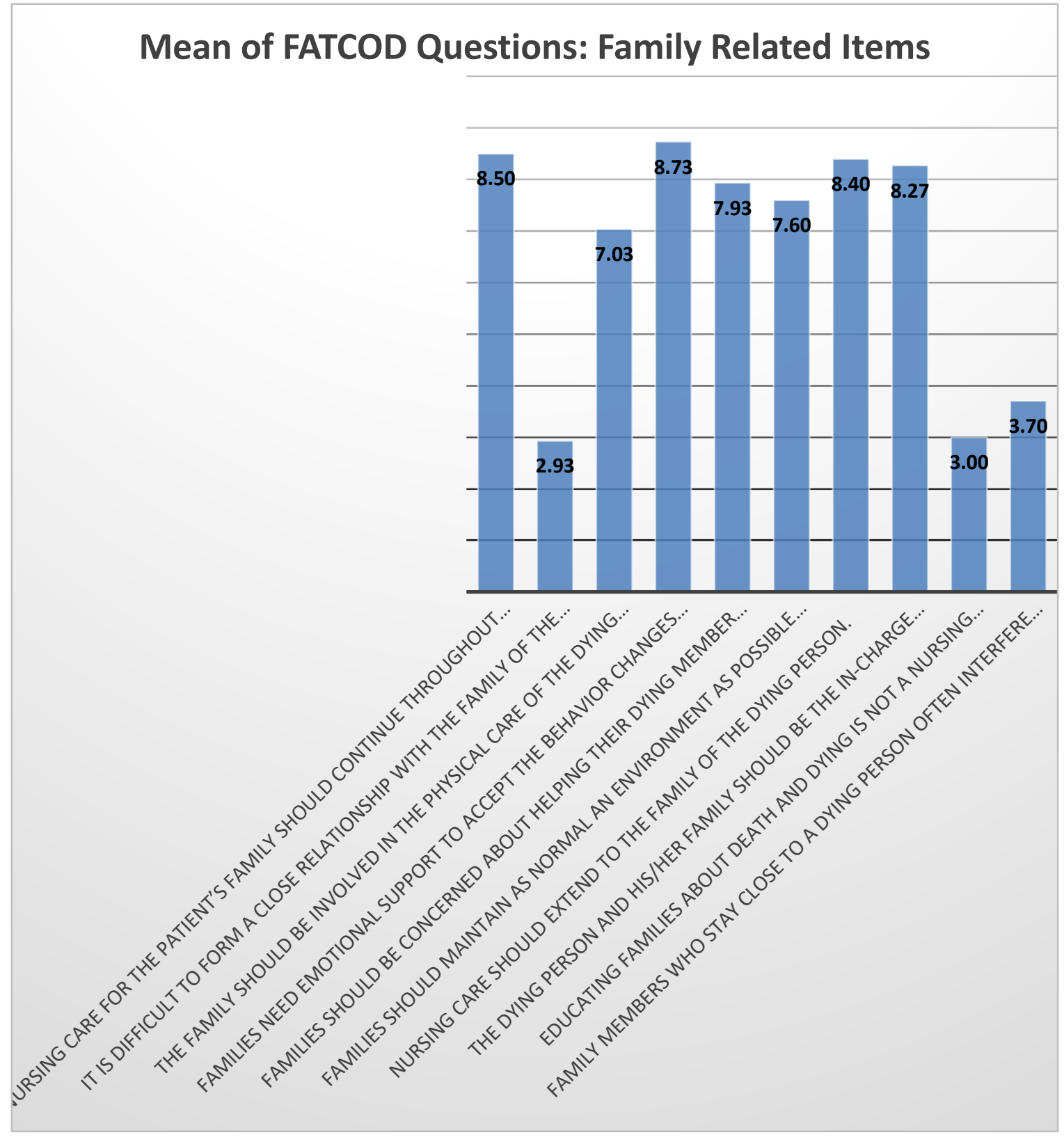


Table 3

\section{FATCOD Questions:'unsure' Responses}

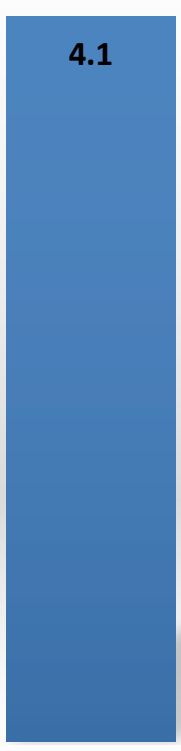

Q2

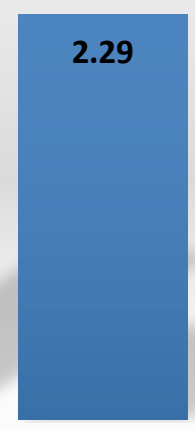

Q3

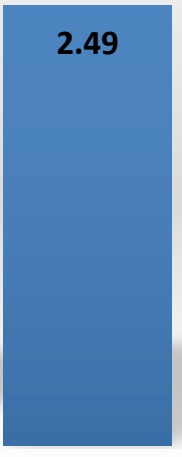

Q6

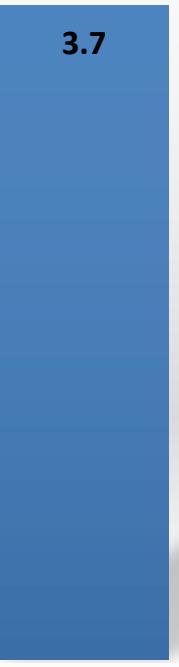

Q12
2.14

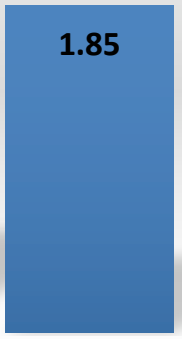

Q8

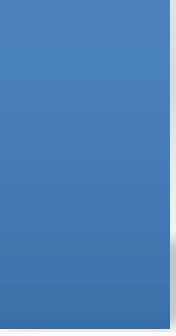

Q13 


\section{Summary and Conclusions}

As palliative care aims to improve the quality of life for patients and their families, the focus on pain and symptom management is an important component in patient centered care. Palliative care specialists are integrated into the interdisciplinary team to coordinate and address complex needs and provide ongoing support to their team members, patients and their caregivers. The National Coalition for Hospice and Palliative Care (NCHPC) defined palliative care as a sub-specialty to provide expert recommendations. Critical care nurses are involved in the changing role of palliative care acute care settings. Approximately one in five deaths in the United States occurs during or shortly after an ICU admission (Angus et al., 2004). To support these patients, the effective integration of palliative care has the potential to guide patients and their families and prepare them for challenges after their hospitalization. One of the important factors discussed is the delivery of palliative care is impacted by the nurses' knowledge, attitudes, beliefs and experiences with end of life, poorly defined attitudes and knowledge may affect the patients' goals and quality of life.

Palliative care is now recognized as a national priority considering the growing number of chronically ill adults are on the rise and frequently require critical care. This change in critical care culture requires nurses to reflect on and embrace palliative care and the provision of high-quality end-of-life care. It is important to identify clinical gaps and gather a needs assessment to prepare and integrate palliative care into their critical care nursing education. The attitudes of the nurses have a direct impact in patient care, and education on end-of-life will assimilate confidence in caring for the dying. The aim 
of this study was to understand the nursing current attitudes towards caring for the dying within a critical care setting.

Critical care nurses play an important role in delivering end-of-life care. The results of this survey indicate that this sample of critical care nurses reported to have a positive attitude towards death and dying, including adequate palliative care knowledge of patients and their families. It is assumed that the high scores on the FATCOD from this study may be due to the nurse's higher level of general knowledge and acceptance of palliative care. Over the past several years, specialized palliative care teams played an important role in this hospital setting and incorporated continuous education with the critical care nurses. Designated palliative care teams provide continuous education through pain and palliative care committee meetings, attending care rounds, posting new clinical resources for pain and symptom management. These additional palliative care resources may have contributed to a positive attitude score. The surveyed nurses were all considered to have a critical care background, no demographic data was collected to maintain confidentiality, therefore years of experience, age and gender may have played an important role in confidence and understanding of how to create a rapport with the families.

The FATCOD survey used in this acute care setting demonstrated high confidence scores in knowledge and attitudes towards caring for the dying patient and their families. The survey suggested that nurses are incorporating families into the patient care and strong advocates for patient-center care. Survey questions with high mean scores are indicative of a positive attitude and confidence in caring for dying patient and their families, while low means were observed with those reporting to have poor knowledge 
and confidence. The survey included patient and family related items to support the overall care of a dying patient. The data was categorized as family related items and patient related items. The patient related items were then categorized into positive and negative statements to correlate an appropriate understanding of the statements.

The highest mean score on the survey was 4.75 . This was categorized in the patient related items and identified the nurses' confidence levels by asking "if nurses should permit a hospice patient with flexible visitation hours" this affirms that this population of nurses have practiced patient center care and autonomy and are comfortable with patient advocacy. The highest mean score for family related items asked nurses "if families need emotional support to accept the behavior changes of the dying person" with a mean score of 4.59. Surveyed nurses all answered with a positive response. This is assumes that nurses are able to provide families with comfort and support. Survey questions with high mean scores was indicative of a positive attitude and confidence in caring for dying patient and their families.

Patient related questions addressed their physical, emotional, psychosocial and spiritual dimensions. Nurses had a positive response rate, $96 \%($ mean $=8.67)$ reported that caring for a dying patient is a worthwhile experience, $100 \%($ mean $=8.67)$ understand the progression of death when nurses are aware that death is welcomed by the dying person, $100 \%($ mean $=8.97)$ believed in the benefits of listening to patients as they verbalize their feelings, $98 \%($ mean $=9.03)$ allowed flexible visitation schedules for dying patients, $89 \%($ mean $=8.43)$ had knowledge in pain management and the role of adequate pain relief, $97 \%($ mean $=8.90)$ of nurses are honest with the patient about their 
condition and $91 \%($ mean $=8.23)$ are assuming the responsibility to help patients prepare for death.

The survey was conducted with positive and negative statements, low means correlated with negative statements, therefore nurses opposed to the statement are identified to have a positive attitude. Nurses felt comfortable being assigned to care for a dying patient, while 4\% was unsure or was not comfortable being assigned to one. Additionally, nurses were asked if patients should not be allowed to make decisions about their physical care, $98 \%($ mean $=2.30)$ of nurses disagreed and rationalized that patients should be involved in their care.

Family related items incorporated the families into the patients plan of care and acknowledges their feelings and effective support from the providers. Nurses responded with a high mean (8.50), 90\% of nurses suggested that nursing care for the patient's family should be continue throughout the period of grief and bereavement. Additionally, $100 \%(8.73)$ was observed that emotional support for families is needed to accept the changes of a dying person.

Overall, the survey indicated a high confidence and positive attitude in both patient and family care areas of inquiry. There are potential areas for improvement in palliative care education indicated by the study findings. Nurses should continue to advocate and deliver optimal end-of-life patient care and reflect on their personal beliefs that may challenge caring for dying patients and their families. Opportunities for future programs were identified from high number of responses of "unsure" answers. Six questions were identified and sorted into categories of weakness and need for further investigation. These answers were identified in areas of critical care nurses experience, 
confidence, communication, belief system, delegation and support, in regards to caring for patients at end of life. 


\section{Recommendations and Implications for Advanced Nursing Practice}

It is important that continuous education and self- reflection is routinely practiced in the advanced practice role. The role of an advanced practitioner includes the treatment plan and supportive counseling for patients and their families. Wiener et al., 2015 detailed the importance of competent, confident and compassionate providers and proposed palliative care educational strategies to promote high-quality patient centered care and comfort in terminally- ill patients. Although critical care nurses have a positive attitude towards caring for dying patients and supporting families, advanced practitioners continue the support with transformational learning traits that drive the patients' treatment plan and guide goals of care conversations. Mezirow's Transformative

Learning Theory helps define the personal challenges of nurses and addresses the basis of ethical dilemmas they encounter. Providing accurate palliative care education to critical care nurses will identify outdated frames of reference and shape the attitudes and beliefs to provide positive measures of self-reflection to improve patient care.

It is recommended that advanced practitioners who are caring for patients with chronic illnesses are able to identify prognostic and predictive factors in order to facilitate appropriate ethical, clinical, and organizational decisions in addition to utilizing resources as efficiently as possible (Ripamonti et al., 2009). The use of prognostic tools is to inform patients and their caregivers about the prognosis while focusing on the efforts of patient centered care; assist providers in clinical decision making in appropriate treatment therapies; understand the risk and benefits of under and overtreatment and an individualized symptom control plan for patients. Further recommendations include improving the quality of life and symptom management that is patient centered care, as 
well as improving communication between the interdisciplinary team, the patient and their families.

It is vital that each discipline have an opportunity to contribute to high-quality palliative care. One essential provider must be identified to manage an effective goals of care plan. A palliative care consultant may be available to provide expert advice. The role of a palliative specialist focuses on the reduction on symptom burden and the improvement in the patients' quality of life. The support from a palliative care consultant can provide symptom control, organization of further treatments and supporting patients and their caregivers. Although these consults have shown positive effects on patient outcomes, limitations include organizational strategies to define policies and procedures, financial barriers and lack of staff availability, knowledge in end-of-life care, and skepticism of the requesting physicians. While a consultation is optional, it is important that providers are aware of their limitations, serve as leaders on the multidisciplinary team and utilize effective resources to achieve optimal goals of care. 


\section{References}

Aldridge, M. D., \& Kelley, A. S. (2015). The myth regarding the high cost of end-oflife care. American journal of public health, 105(12), 2411-2415. https://doi.org/10.2105/AJPH.2015.302889

Ashley, J. L., \& Fasolino, T. K. (2016). Palliative and hospice care: Educational needs of inpatient registered nurses. Creative Nursing,22(2), 114-120. doi:10.1891/1078-4535.22.2.114

Aslakson, R. A., Curtis, J. R., \& Nelson, J. E. (2014). The changing role of palliative care in the ICU. Critical Care Medicine,42(11), 2418-2428. doi:10.1097/ccm.0000000000000573

Anderson, W. G., Puntillo, K., Boyle, D., Barbour, S., Turner, K., Cimino, J., . . . Pantilat, S. (2016). ICU Bedside nurses involvement in palliative care communication: A multicenter survey. Journal of Pain and Symptom Management,51(3). doi:10.1016/j.jpainsymman.2015.11.003

Angus DC, Barnato AE, Linde-Zwirble WT, et al. Robert Wood Johnson Foundation ICU End-of-life peer group: Use of intensive care at the end of life in the United States: An epidemiologic study. Crit Care Med. 2004; 32:638-643. [PubMed: 15090940]

Ayed, A., Sayej, S., MD, Harazneh, L., Fashafsheh, I., MD, \& Eqtait, F. (2015). The Nurses' knowledge and attitudes towards the palliative care. Journal of Education and Practice, 6. Retrieved from www.iiste.org.

Baggs, J. G., Norton, S. A., Schmitt, M. H., \& Sellers, C. R. (2004). The dying patient in the ICU: Role of the interdisciplinary team. Critical Care Clinics, 20(3), 525-540. doi:10.1016/j.ccc.2004.03.008

Cabaniss, R. (2015). Using transformative learning theory to impact patient care. Journal of Nursing \& Care,04(01). doi:10.4172/2167-1168.1000223

Centers for Disease Control and Prevention (2015). Chronic disease overview. Retrieved from http://www.cdc.gov/chronicdisease/overview/

Chang, H.-J., Hsu, L.-L., Hsieh, S.-I., Chu, T.-L., \& Yu, W.-P. (2016). Development of a needs scale for nurses receiving in-service palliative care education. Cancer Nursing, 39(3). doi: 10.1097/ncc.0000000000000277

Cranton, P. (1997). Transformative learning in action: insights from practice. San Francisco: Jossey-Bass.

Dudgeon, D. J., Knott, C., Chapman, C., Coulson, K., Jeffery, E., Preston, S., Smith, A. 
(2009). Development, implementation, and process evaluation of a regional palliative care quality improvement project. Journal of Pain and Symptom Management,38(4), 483-495. doi:10.1016/j.jpainsymman.2008.12.006

Dumanovsky, T., Augustin, R., Rogers, M., Lettang, K., Meier, D. E., \& Morrison, R. S. (2016). The growth of palliative care in U.S. hospitals: A status report. Journal of Palliative Medicine,19(1), 8-15. doi:10.1089/jpm.2015.0351

Ferrell, B. R., Virani, R., Paice, J. A., \& Malloy, P. (2010, December 01). Betty R. Ferrell. Retrieved from http://ccn.aacnjournals.org/content/30/6/40.short

Grant, M., Elk, R., Ferrell, B., Morrison, R. S., \& Gunten, C. F. (2009). Current status of palliative care--clinical implementation, education, and research. CA: A Cancer Journal for Clinicians, 59(5), 327-335. doi:10.3322/caac.20032

Harden, K., Price, D., Duffy, E., Galunas, L., \& Rodgers, C. (2017). Palliative care: improving nursing knowledge, attitudes, and behaviors. Clinical Journal of Oncology Nursing,21(5). doi:10.1188/17.cjon.e232-e238

Institute of Medicine (IOM). (2014). Dying in America: improving quality and honoring individual preferences near the end of life. Washington, DC: The National Academies Press.

Jenkins, B., \& Warren, N. A. (2012). Critical Care Nursing Quarterly, 35(4), 388-395. doi: 10.1097/cnq.0b013e318268fe09

Lilly CM, De Meo DL, Sonna LA, Haley KJ, Massaro AF, Wallace RF, et al. An intensive communication intervention for the critically ill patient. Crit Care Clin 2002;18(3): 659-81

Manfredi, P. I., Morrison, R. S., Morris, J., Goldhirsch, S. I., Carter, J. M., \& Meier, D. I. (2000). Palliative care consultations: How do they impact the care of hospitalized patients? Journal of Pain and Symptom Management, 20(3), 166-174.

McEwen, M., \& Wills, E. (2014). Theoretical basis for nursing ( $4^{\text {th }}$ ed). Philadelphia: Wolters Kluwer.

Mitchell G. K. (2011). Palliative care in Australia. The Ochsner journal, 11(4), 334-337.

National Center for Health Statistics, Centers for Disease Control and Prevention. (2013) Summary health statistics for the U.S. population: National health interview Survey, 2012. Accessed at http://www.cdc.gov/nchs/data/series/sr_10/sr10_259.pdf 
National Consensus Project for Quality Palliative Care (NCP). (2009). Clinical Practice Guidelines for Quality Palliative Care. ( $2^{\text {nd }}$ ed.). Retrieved May 3, 2019, from: http://www.nationalconsensusproject.org/guideline.pdf

Nelson, J. E., Cortez, T. B., Curtis, J. R., Lustbader, D. R., Mosenthal, A. C., Mulkerin, C,. (2011). Integrating palliative care in the ICU: The nurse in a leading role. Retrieved from https://www.ncbi.nlm.nih.gov/pmc/articles/PMC3162372/

Polit, D. \& Beck, C. T. (2017). Nursing research: Generating and assessing evidence for nursing practice (10th ed.). Philadelphia: Wolters Kluwer.

Puntillo KA. The role of critical care nurses in providing and managing end-of-life care. In: Curtis JR, Rubenfeld GD, editors. Managing death in the ICU: the transition from cure to comfort. New York: Oxford University Press; 2001. P 149-64

Renigere, R. (2014). Transformative learning in the discipline of nursing. American Journal of Educational Research,2(12), 1207-1210. doi:10.12691/education-2-1212

Ripamonti, C. I., Farina, G., \& Garassino, M. C. (2009). Predictive models in palliative care. Cancer, 115(S13), 3128-3134. doi: 10.1002/cncr.24351

Ross, M. M., Mcdonald, B., \& Mcguinness, J. (1996). The palliative care quiz for nursing (PCQN): The development of an instrument to measure nurses knowledge of palliative care. Journal of Advanced Nursing,23(1), 126-137. doi:10.1111/j.13652648.1996.tb03106.x

Sekse, R. J. T., Hunskår, I., \& Ellingsen, S. (2017, July 11). The nurse's role in palliative care: A qualitative meta-synthesis. Retrieved from https://onlinelibrary.wiley.com/doi/abs/10.1111/jocn.13912.

Sebastiano Mercadante, Cesare Gregoretti, \& Andrea Cortegiani. (2018, August 16). Palliative care in intensive care units: Why, where, what, who, when, how. Retrieved from https://bmcanesthesiol.biomedcentral.com/articles/10.1186/s12871-018-0574-9

Skar, R. Knowledge use in nursing practice: The importance of practical understanding and personal involvement. Nurse Educ Today 2010;30:132-6.

Schmitt MH. Collaboration improves the quality of care: methodological challenges and evidence from US health care research. J Interprof Care 2001; 15(1):47-66

Tait, V., Higgs, M., Magann, L., Dixon, J., Davis, J. M., \& Fernandez, R. (2015). Attitudes of nonpalliative care nurses towards palliative care. International Journal of Palliative Care, 2015, 1-6. doi: 10.1155/2015/469174 
Wiener, L., Weaver, M. S., Daly, U. M., \& Bell, C. J. (2015). Threading the cloak: palliative care education for care providers of adolescents and young adults with cancer. Clinical Oncology in Adolescents and Young Adults, 1. doi:10.2147/coaya.s49176

Wittenberg-Lyles, E., Goldsmith, J., \& Ragan, S. (2011). The shift to early palliative care. Clinical Journal of Oncology Nursing, 15(3), 304-310. doi:10.1188/11.cjon.304-310

World Health Organization. (2015). Palliative Care. Retrieved from http://www.who.int/meddiacentre/factsheets/fs402/en

Yorks, L., \& Sharoff, L. (2001). An extended epistemology for fostering transformative learning in holistic nursing education and practice. Holistic Nursing Practice, 16(1), 21-29. doi:10.1097/00004650-200110000-00007 


\section{Appendix A}

\section{FROMMELT ATTITUDES TOWARD CARE OF THE DYING SCALE}

Instructions: Please circle the letter following each statement which corresponds to your own personal feelings about the attitude or situation presented. Please respond to all 30 statements on the scale. The meanings of the letters are:

$\mathrm{SD}=$ Strongly Disagree $\quad \mathrm{D}=$ Disagree $\quad \mathrm{U}=$ Uncertain $\mathrm{A}=$ Agree $\quad \mathrm{SA}=$ Strongly Agree

1. Giving nursing care to the dying person is a worthwhile learning experience.
SD D U A $\quad$ SA

2. Death is not the worst thing that can happen to a person.

SD $\quad \mathrm{D} \quad \mathrm{U} \quad \mathrm{A} \quad \mathrm{SA}$

3. I would be uncomfortable talking about impending death with the dying person.

SD $\quad \mathrm{D} \quad \mathrm{U} \quad \mathrm{A} \quad \mathrm{SA}$

4. Nursing care for the patient's family should continue throughout the period of grief and bereavement.

SD D U A $\quad$ SA

5. I would not want to be assigned to care for a dying person.

SD D U A SA

6. The nurse should not be the one to talk about death with the dying person.

SD D U A $\quad$ SA

7. The length of time required to given nursing care to a dying person would frustrate me.

SD D U A $\quad$ SA

8. I would be upset when the dying person I was caring for gave up hope of getting better.

SD D U A SA

9. It is difficult to form a close relationship with the family of the dying person.

SD D U A SA

10. There are times when death is welcomed by the dying person.

SD D U A SA 
11. When a patient asks, "Nurse am I dying?" I think it is best to change the subject to something cheerful.
SD D U A SA

12. The family should be involved in the physical care of the dying person.

SD D U A SA

13. I would hope the person I'm caring for dies when I am not present.

\section{SD $\quad \mathrm{D} \quad \mathrm{U} \quad \mathrm{A} \quad \mathrm{SA}$}

14. I am afraid to become friends with a dying person.

SD D U A SA

15. I would feel like running away when the person actually died.

SD D U A SA

16. Families need emotional support to accept the behavior changes of the dying person.

SD $\quad \mathrm{D} \quad \mathrm{U} \quad \mathrm{A} \quad \mathrm{SA}$

17. As a patient nears death, the nurse should withdraw from his/her involvement with the patient.

$$
\text { SD } \quad \mathrm{D} \quad \mathrm{U} \quad \mathrm{A} \quad \mathrm{SA}
$$

18. Families should be concerned about helping their dying member make the best of his/her remaining life.

$$
\text { SD D U A } \quad \text { SA }
$$

19. The dying person should not be allowed to make decisions about his/her physical care.

$$
\text { SD D U A SA }
$$

20. Families should maintain as normal an environment as possible for their dying member.

$$
\text { SD D U A SA }
$$

21. It is beneficial for the dying person to verbalize his/her feelings.
SD D
$\mathrm{U}$
A
SA

22. Nursing care should extend to the family of the dying person. 
SD D U A SA

23. Nurses should permit dying persons to have flexible visiting schedules.

$$
\text { SD D U A SA }
$$

24. The dying person and his/her family should be the in-charge decision makers.

SD D U A SA

25. Addiction to pain relieving medication should not be a concern when dealing with a dying person.

$$
\text { SD D U A SA }
$$

26. I would be uncomfortable if I entered the room of a terminally ill person and found him/her crying.

$$
\text { SD D U A SA }
$$

27. Dying persons should be given honest answers about their condition.

$$
\text { SD D U A SA }
$$

28. Educating families about death and dying is not a nursing responsibility.

$$
\text { SD D U A } \quad \text { SA }
$$

29. Family members who stay close to a dying person often interfere with the professionals' job with the patient.
SD D U A SA

30. It is possible for nurses to help patients prepare for death.

SD D U A $\quad$ SA

Copywrite 1988, Katherine H. Murray Frommelt 


\title{
Appendix B
}

\section{Consent to Participate in a Needs Assessment}

\section{Critical Care Nurses' Attitudes Toward the Care of the Dying}

\author{
Researcher: Ny Tran, BSN, RN
}

\section{What is the research about and why are you doing it?}

This research is being conducted to obtain information on critical care nurses' attitudes toward care of the dying. The information will be used to provide recommendations on ways to educate nurses on care of the dying patient.

\section{What are you asking me to do if I agree to be in the study?}

If you agree to participate in the study, you will complete one survey. The surveys will be available on your unit for two weeks. Each survey consists of 30 questions; you will rate each question from strongly disagree to strongly agree, depending on your opinions.

\section{How will this study help me?}

The information obtained from this study may not help you. However, it may help others by making recommendations for education on care of the dying patient.

\section{Are there any risks involved with being in the study?}

There are no anticipated risks or harms to you as a result of your participation in the study. Although, answering questions on care of the dying patient may bring on feelings of sadness or anxiety. Participation in the study will be anonymous, voluntary, and will not affect employment status in any way.

\section{What steps have been taken to minimize participant risk?}

Your identity will be anonymous. No identifying demographics will be obtained.

\section{What else do I need to know?}

Your decision to participate is completely voluntary.

\section{Whom can I contact with any questions or concerns?}

If you have any questions, you may contact Ny Tran at (774) 365-0976. If you have concerns about this study, please contact the Institutional Review Board.

Results of the study will be available after June 2020 . 
***By completing this survey, you are giving your consent for participation in the research study. 